\title{
Drug Utilization Patterns in Patients with Diabetes Initiating Sodium Glucose Co-Transporter-2 Inhibitors (SGLT2i) in Japan: A Multi-Database Study (2014-2017)
}

Yuichiro Ito · James Van Schyndle - Takuya Nishimura •

Toshifumi Sugitani · Tomomi Kimura (D)

Received: August 30, 2019 / Published online: October 18, 2019

(C) The Author(s) 2019

\begin{abstract}
Introduction: This study aimed to identify drug utilization patterns in patients initiating sodium glucose co-transporter-2 inhibitors (SGLT2i) in the first 3 years of their launch in Japan.

Methods: This was a retrospective study using three administrative databases in Japan: a pharmacy claims database, a hospital-based database, and an insurance claims database. Prescription data were extracted from adult outpatients with diabetes who started SGLT2i between April 2014 and March 2017 to evaluate pre-index and concomitant medications. For glimepiride and insulin co-users, dose at SGLT2i add-on was also assessed.
\end{abstract}

Enhanced Digital Features To view enhanced digital features for this article go to https://doi.org/10.6084/ m9.figshare.9918941.

Electronic Supplementary Material The online version of this article (https://doi.org/10.1007/s13300019-00710-2) contains supplementary material, which is available to authorized users.

Y. Ito · T. Nishimura · T. Sugitani · T. Kimura $(\bowtie)$ Astellas Pharma Inc., 5-1, Nihonbashi-Honcho, Chuo-ku, Tokyo 103-8411, Japan

e-mail: tomomi.kimura@astellas.com

J. Van Schyndle

Astellas US, LLC., 1 Astellas Way, Northbrook, IL 60062-6111, USA
Results: Data from a total of 14,861 patients in the pharmacy dataset (P-dataset), 27,039 in the hospital dataset (H-dataset), and 12,408 in the insurance dataset (I-dataset) were analyzed. The majority of SGLT2i new users (ca. 70\%) were taking one to three concomitant antidiabetic medications. Around half of SGLT2i initiators used dipeptidyl peptidase 4 inhibitors and/or biguanides before using SGLT2i or concomitantly with SGLT2i. The average daily glimepiride dose decreased from $2.1 \mathrm{mg} /$ day during the pre-index period to $1.8 \mathrm{mg} /$ day at SGLT2 $\mathrm{i}$ add-on in the P-dataset and from 1.9 to $1.7 \mathrm{mg} /$ day in the both $\mathrm{H}$ - and I-datasets, respectively, with a decreasing trend observed during the first 3 years of launch. The average daily insulin dose at SGLT2i add-on was higher during the first 15 months of launch and then decreased thereafter. Nearly $40 \%$ or more SGLT2i new users were taking at least five concomitant medications: cardiovascular agents were predominantly co-prescribed.

Conclusion: SGLT2i were frequently used as second- or later-line treatment and as part of a dual, triple, or quadruple regimen, as well as coprescribed with many other medications in the first 3 years of their launch. For SGLT2i users taking concomitant SU or insulin medications, the average daily doses of SU and insulin at SGLT2i add-on decreased slightly over the study period.

Funding: Astellas Pharma Inc., Tokyo, Japan. 
Keywords: Administrative databases; Initiate; Japan; Sodium glucose co-transporter-2 inhibitors; Utilization pattern

\section{INTRODUCTION}

The prevalence of diabetes in Japan is projected to reach $9.8 \%$ by 2030 and affect 9.7 million people [1]. Type 2 diabetes mellitus (T2DM) accounts for around $90 \%$ of patients with diabetes and is a global public health concern [2]. T2DM and its associated complications have a detrimental impact on patients' quality of life and place an enormous burden on the patients, family, and society [2].

Sodium glucose co-transporter-2 inhibitors (SGLT2i) are a new class of oral antidiabetic drugs (OADs), which operate independently of insulin action and are not affected by the degree of insulin resistance or level of $\beta$-cell function [3]. SGLT2i reduce blood glucose by inhibiting the renal reuptake of glucose and promoting urinary glucose excretion, with a consequent loss of calories and reduced body weight [3-5]. Six SGLT2i compounds, ipragliflozin, dapagliflozin, luseogliflozin, tofogliflozin, canagliflozin, and empagliflozin (in order of date of launch), were introduced in Japan between April 2014 and February 2015 for use as monotherapy or as add-on to other antidiabetic medications in adults with T2DM [6-14]. A recent systematic review and meta-analysis of clinical trials of SGLT2i in patients with T2DM reported that SGLT2i reduce hospitalization for heart failure and progression of renal disease [15].

In patients with T2DM who failed to achieve target glycated hemoglobin (HbA1c) with lifestyle modification and first-line agent, combination therapy with another antidiabetic agent with a different mechanism of action is usually recommended [16-18]. International guidelines recommend to consider SGLT2i as one of the options for second-line therapy in patients with T2DM who have chronic cardiovascular disease or chronic kidney disease, or in those who have compelling needs to minimize hypoglycemia or weight gain [16, 17], whereas the Japan Diabetes Society recommends that the choice of antidiabetic agents should be based on the disease condition of individual patients with consideration given to the pharmacological and safety profile of each antidiabetic agent [18]. In order to manage the potential risk of hypoglycemia, the package inserts for SGLT2i state that the dose for sulfonylureas (SU) or insulin should be reduced when adding SGLT2i to either of these agents [19-24]. It is essential to reduce the insulin dose cautiously, especially in patients with decreased insulin secretion to minimize the risk of ketoacidosis [25]. Also, for patients on diuretics, physicians are advised to take into consideration the diuretic effects of SGLT2i when starting treatment to avoid excessive fluid loss [19-24].

Previous studies have reported the prescription patterns of OADs [26-29], but relevant data on SGLT2i as a class are still limited in Japan [30], especially for details on concomitant medications, such as SU or insulin dose at SGLT2i add-on. We previously reported the characteristics of SGLT2i new users who tended to be younger and have a higher prevalence of hypercholesterolemia, a higher body mass index, and a higher HbA1c level than new users of other OADs [31]. Here, we further examined pre-index and concomitant medication use in SGLT2i new users in the first 3 years of SGLT2i launch in Japan.

\section{METHODS}

This was a retrospective, cross-sectional analysis using three distinct Japanese administrative databases to characterize drug utilization patterns in SGLT2i new users. The study protocol was approved by the Medical Affairs Japan Protocol Review Committee on 22 June 2017 before study commencement. As the study only involved analysis of pre-existing data from the data sources and the data were anonymized, written informed consent was not required, according to applicable regulations in Japan $[32,33]$. This study was conducted in accordance with the guidelines for Good Pharmacoepidemiology Practice [34]. 


\section{Data Sources}

Three data sources were used: (1) a pharmacy claims database provided by Kyowa Kikaku Ltd. consisting of dispensing claims from over 800 panel pharmacies nationwide, which provided a coverage of approximately $2 \%$ of all out-ofhospital dispensing claims [35]; (2) a hospitalbased database provided by Medical Data Vision Co. Ltd. including medical and pharmacy claims from 287 Diagnosis Procedure Combination (DPC) hospitals nationwide (at the time of the study) from more than 17 million outpatients and inpatients [36]; and (3) an insurance claims database provided by JMDC Inc., containing medical and prescription claims of 3.8 million employees and their dependents who were mostly aged 65 years or less $[37,38]$.
Details of these databases were described elsewhere [39].

\section{Study Population}

The study population was SGLT2i new users who were identified using the methods previously described [31]. The patient flow is presented in Fig. 1. Briefly, three separate study datasets were developed from each database: pharmacy dataset (P-dataset; from pharmacy claims database), hospital dataset (H-dataset; from hospital-based database), and insurance dataset (I-dataset; insurance claims database). The P-dataset was considered as the primary dataset because it included data from both small clinics and large hospitals, and had no limitation in patients' age. The H-dataset was used because it was one of the largest commercially

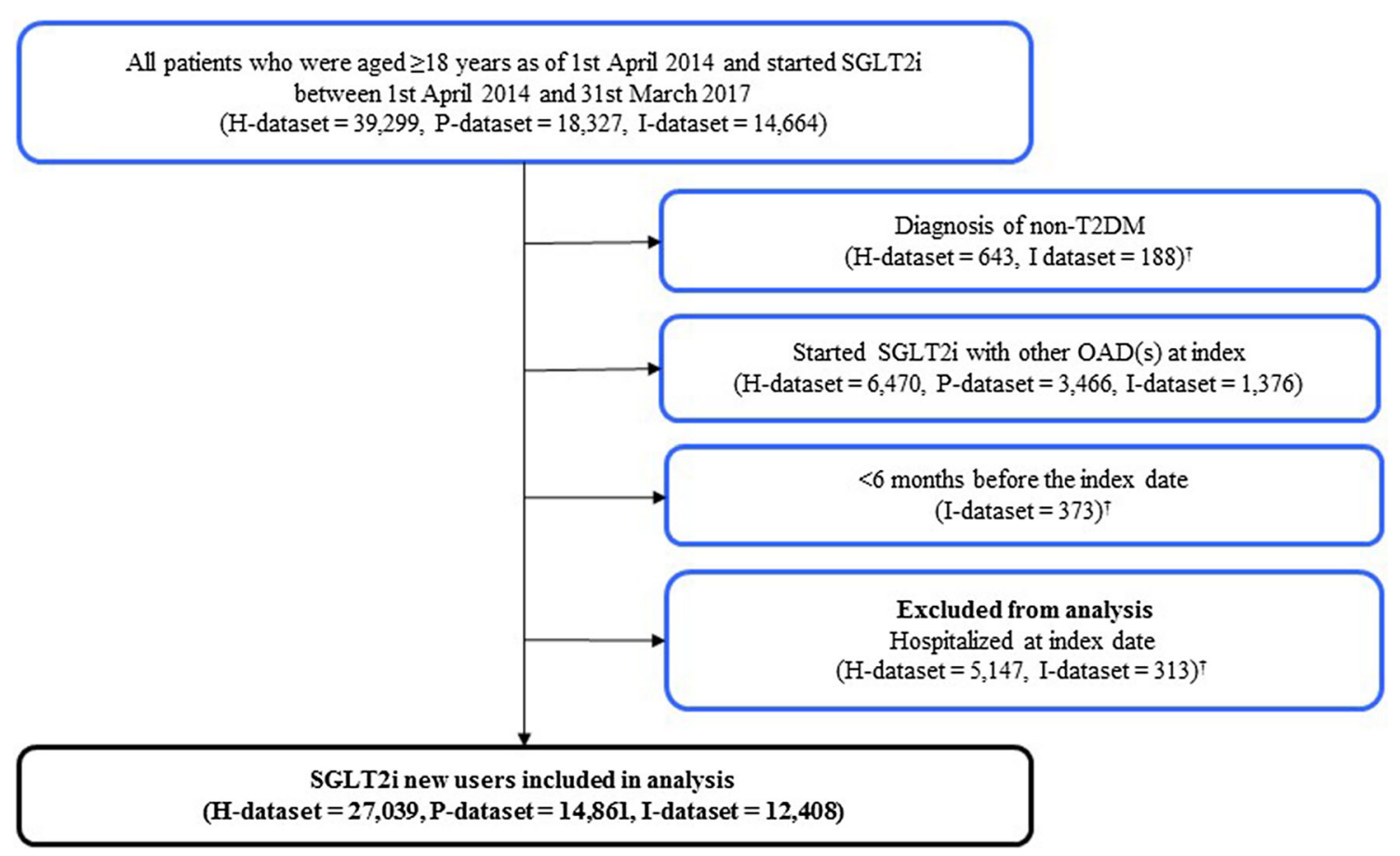

Fig. 1 Patient flow for SGLT2i new users. As a result of the different nature of the datasets, certain exclusion criteria were only applicable to the datasets indicated by a dagger. P-dataset: From a pharmacy claims database using data from over 800 pharmacies nationwide which provided a coverage of approximately $2 \%$ of all outpatient prescriptions. H-dataset: From a hospital-based administrative database constructed from data for inpatients and outpatients from 287 Diagnosis Procedure Combination (DPC) hospitals. I-dataset: From an insurance claims database containing medical and prescription claims of 3.8 million employees and their dependents who were mostly aged 65 years or less. FDC fixed-dose combination, non-T2DM non-type 2 diabetes mellitus, OADs oral antidiabetic drugs, SGLT2i sodium glucose co-transporter2 inhibitors 
available data sources in Japan: no significant differences in patients' characteristics were identified between the $\mathrm{H}$-dataset and P-dataset in our previous analysis [31]. The I-dataset was used because of its complete follow-up ability for the patients. Each dataset included all patients who were aged at least 18 years as of 1 April 2014 and started SGLT2i between 1 April 2014 and 31 March 2017 (study period). Patients who met any of the following criteria were excluded: (1) diagnosis of diabetes other than T2DM (only applicable to the $\mathrm{H}$ - and I-datasets); (2) started SGLT2i together with other OADs at the index date; and (3) did not have a 6-month enrollment period prior to the index date (only applicable to the I-dataset). The index date was defined as the first prescription date of any SGLT2i product. Patients who were hospitalized at the index date were excluded from the analysis for better generalizability (only applicable to the H- and I-datasets).
Two subcohorts were further derived from each study dataset. One was the SU subcohort, including patients who used SU during the preindex period and continued the same subclass of SU (either glimepiride, glibenclamide, or gliclazide) at the index date (i.e., SGLT2i was added to SU). Because more than $80 \%$ of such patients used glimepiride, only data for the glimepiride subcohort is presented. The other was the insulin subcohort, including patients who used insulin during the pre-index period and on the index date, plus at least one post-index insulin prescription within 3 months from the index date.

\section{Study Assessments}

Pre-index and concomitant antidiabetic medications, and other concomitant medications, including diuretics, were assessed. Antidiabetic

Table 1 Demographics and prescription information for SGLT2i new users

\begin{tabular}{lccc}
\hline & P-dataset $(\boldsymbol{N}=\mathbf{1 4 , 8 6 1})$ & H-dataset $(\boldsymbol{N}=\mathbf{2 7 , 0 3 9})$ & I-dataset $(\boldsymbol{N}=\mathbf{1 2 , 4 0 8})$ \\
\hline Median age, years $(\mathrm{IQR})$ & $59(50-68)$ & $59(49-67)$ & $52(46-58)$ \\
Gender, male, $n(\%)$ & $9212(62.0)$ & $16,839(62.3)$ & $9073(73.1)$
\end{tabular}

Number of pre-index antidiabetic medications

$\begin{array}{lccr}\text { Mean }(\mathrm{SD}) & 2.1(1.3) & 2.3(1.3) & 2.2(1.3) \\ \text { Median }(\mathrm{IQR}) & 2(1-3) & 2(1-3) & 2(1-3)\end{array}$

Number of concomitant antidiabetic medications (by class)

$\begin{array}{lrrr}\text { Mean }(\mathrm{SD}) & 1.7(1.3) & 2.0(1.3) & 1.8(1.3) \\ \text { Median (IQR) } & 2(1-3) & 2(1-3) & 2(1-3)\end{array}$

Number of any concomitant medication (by product) ${ }^{\mathrm{a}}$

$\begin{array}{lrrr}\text { Mean }(\mathrm{SD}) & 4.8(3.3) & 5.4(3.5) & 4.1(2.7) \\ \text { Median }(\mathrm{IQR}) & 4(2-7) & 5(3-7) & 4(2-6)\end{array}$

P-dataset: From a pharmacy claims database using data from over 800 pharmacies nationwide which provided a coverage of approximately $2 \%$ of all outpatient prescriptions

H-dataset: From a hospital-based administrative database constructed from data for inpatients and outpatients from 287 Diagnosis Procedure Combination (DPC) hospitals

I-dataset: From an insurance claims database containing medical and prescription claims of 3.8 million employees and their dependents that were mostly aged $\leq 65$ years

$I Q R$ interquartile range, $S D$ standard deviation, $S G L T 2 i$ sodium glucose co-transporter-2 inhibitors

${ }^{a}$ Based on Ministry of Health, Labour and Welfare drug classification [40] 


\section{$\mathbf{a}$}

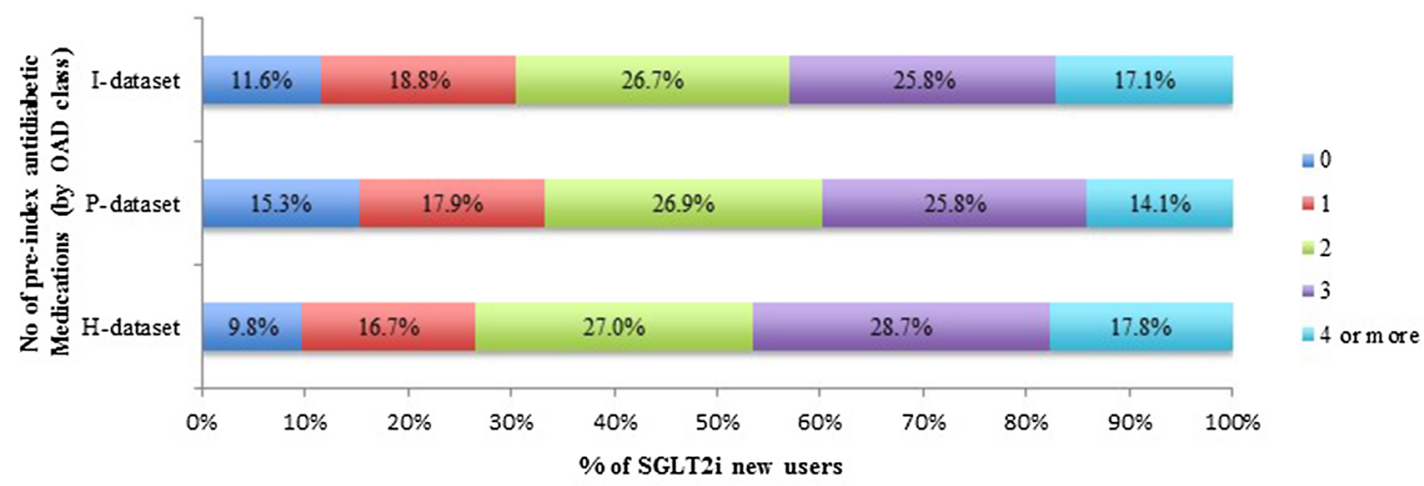

b - I-dataset $\quad \equiv$ P-dataset $\equiv$ H-dataset

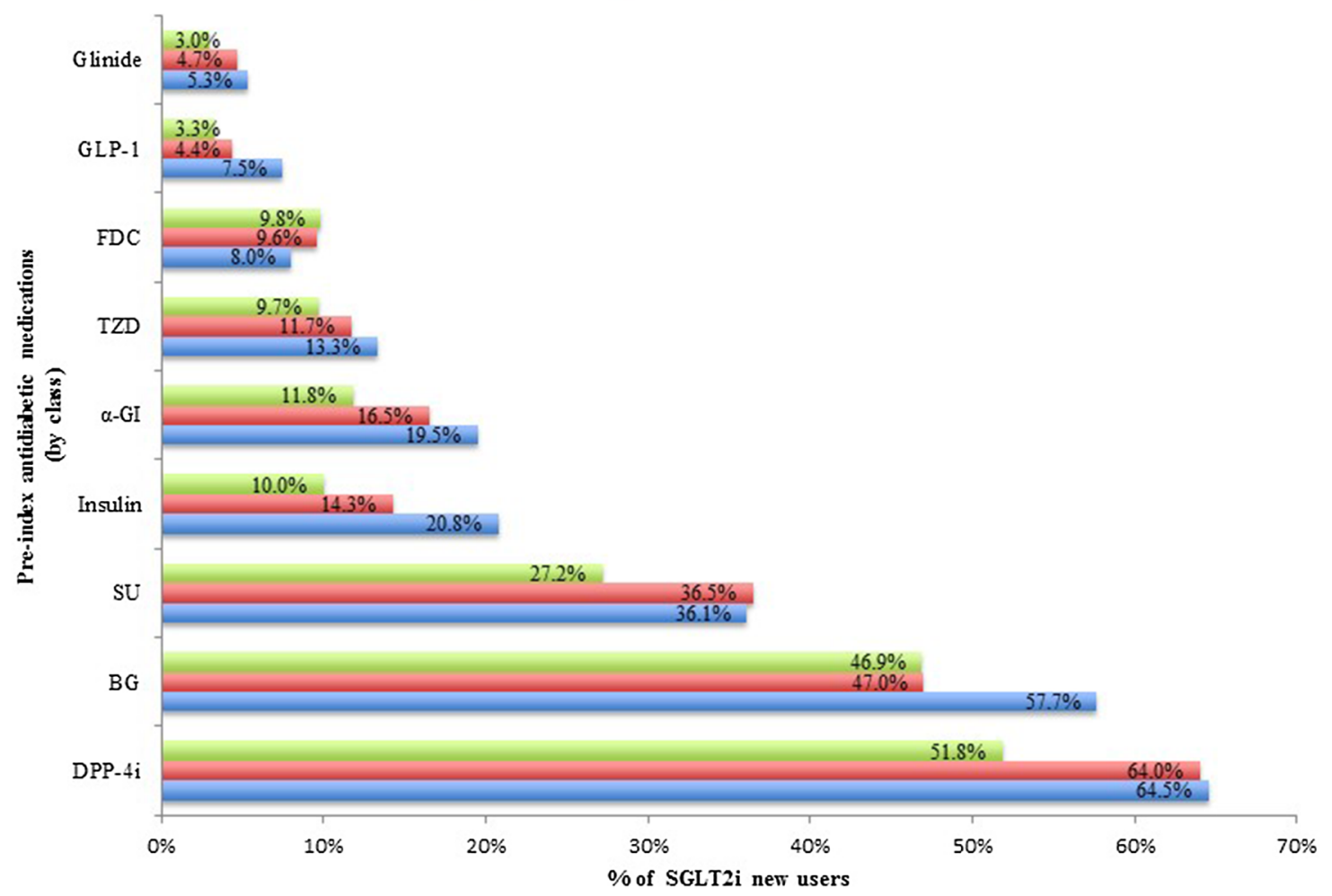

Fig. 2 Distribution of the $\mathbf{a}$ number and $\mathbf{b}$ class of preindex antidiabetic medications. P-dataset: From a pharmacy claims database using data from over 800 pharmacies nationwide which provided a coverage of approximately $2 \%$ of all outpatient prescriptions. H-dataset: From a hospital-based administrative database constructed from data for inpatients and outpatients from 287 Diagnosis Procedure Combination (DPC) hospitals. I-dataset: From an insurance claims database containing medical and prescription claims of 3.8 million employees and their dependents who were mostly aged 65 years or less. $\alpha-G I$ alpha-glucosidase inhibitors, BG biguanides, DPP4i dipeptidyl peptidase 4 inhibitors, FDC fixed-dose combination, GLP-1 glucagon-like peptide 1, OADs oral antidiabetic drugs, SGLT2i sodium glucose co-transporter-2 inhibitors, SU sulfonylureas, TZD thiazolidinediones 


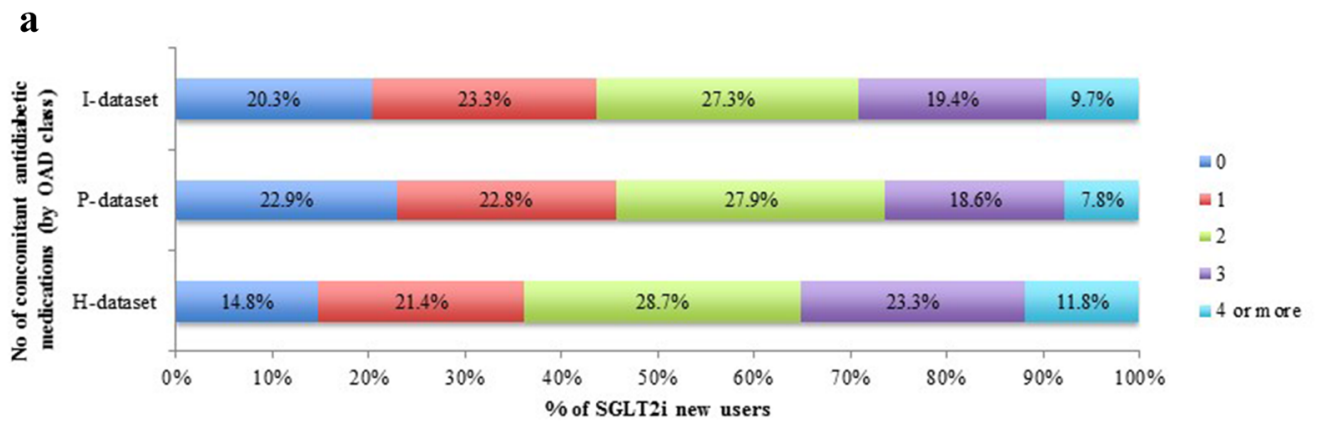

b

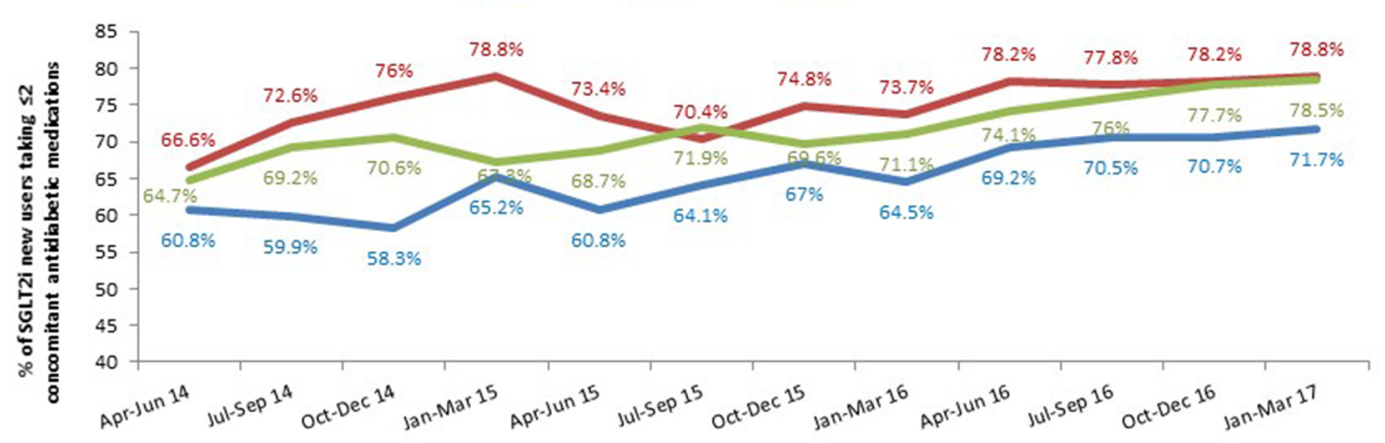

\begin{tabular}{|c|c|c|c|c|c|c|c|c|c|c|c|c|}
\hline & Apr-Jun 14 & Jul-Sep 14 & Oct-Dec 14 & Jan-Mar 15 & Apr-Jun 15 & Jul-Sep 15 & Oct-Dec 15 & Jan-Mar 16 & Apr-Jun 16 & Jul-Sep 16 & Oct-Dec 16 & Jan-Mar 17 \\
\hline $\mathrm{H}$-dataset (n) & 618 & 813 & 629 & 534 & 1831 & 2325 & 3159 & 3444 & 3242 & 2876 & 3523 & 4045 \\
\hline P-dataset (n) & 1133 & 861 & 529 & 462 & 1117 & 1103 & 1445 & 1520 & 1593 & 1521 & 1744 & 1833 \\
\hline 1-dataset (n) & 1162 & 715 & 449 & 370 & 1115 & 1154 & 1196 & 1239 & 1193 & 1187 & 1244 & 1384 \\
\hline
\end{tabular}

c

I-dataset $=$ P-dataset $=$ H-dataset

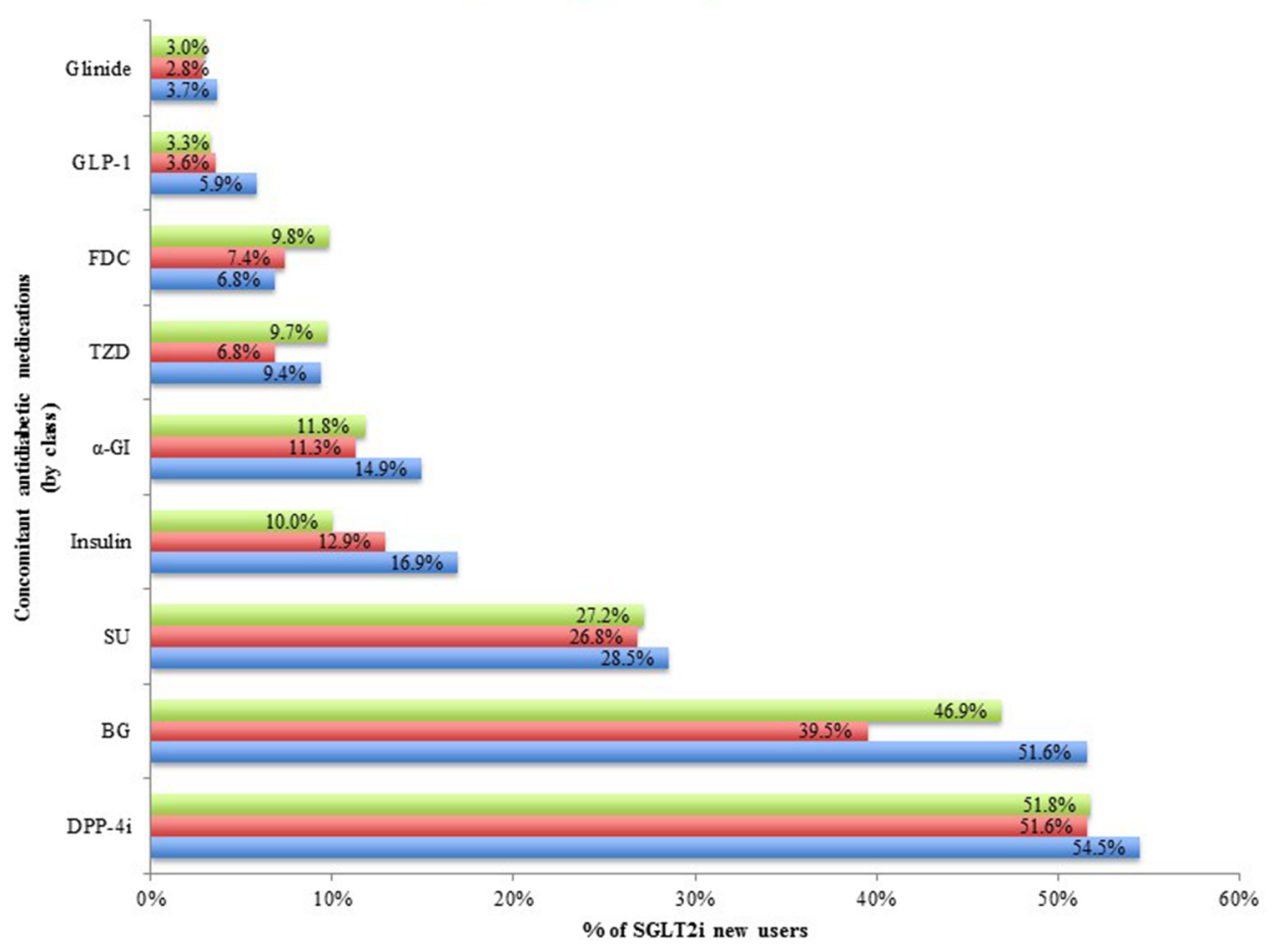


4Fig. 3 a Distribution of the number of concomitant antidiabetic medications. b Chronological change in the proportion of SGLT2i new users taking two or fewer concomitant antidiabetic medications at index. c Distribution of the class of concomitant antidiabetic medications. P-dataset: From a pharmacy claims database using data from over 800 pharmacies nationwide which provided a coverage of approximately $2 \%$ of all outpatient prescriptions. H-dataset: From a hospital-based administrative database constructed from data for inpatients and outpatients from 287 Diagnosis Procedure Combination (DPC) hospitals. I-dataset: From an insurance claims database containing medical and prescription claims of 3.8 million employees and their dependents who were mostly aged 65 years or less. $\alpha$-GI alpha-glucosidase inhibitors, BG biguanides, DPP4i dipeptidyl peptidase 4 inhibitors, FDC fixed-dose combination, GLP-1 glucagon-like peptide 1, OADs oral antidiabetic drugs, SGLT2i sodium glucose cotransporter-2 inhibitors, SU sulfonylureas, TZD thiazolidinediones

medications were categorized by class: alphaglucosidase inhibitors (alpha-GI), biguanides (BG), dipeptidyl peptidase 4 inhibitors (DPP4i), glinides, SU, and thiazolidinediones (TZD), fixed-dose combination of OADs (FDC) and injectable treatment (insulin and glucagon-like peptide 1 [GLP-1] receptor agonists). Concomitant medications were categorized according to the Ministry of Health, Labour and Welfare (MHLW) drug classification [40]. The number of concomitant antidiabetic medications was counted by class, whereas the number of any concomitant medication was counted by product.

In the SU subcohort, the daily dose of SU at SGLT2i add-on (i.e., at the index date) was calculated by multiplying daily number of pills by the dose strength (milligrams) at the index date. The daily dose of SU during the pre-index period was calculated as the average daily dose of SU during that period. In the insulin subcohort, the daily insulin dose (units) at the index date was computed as the average daily dose during the interval from the index date to the next insulin prescription because the intended daily dose could not be obtained directly from the prescription. The pre-index daily dose of insulin was estimated as the average daily dose of insulin during the 180 days prior to the index date.

\section{Statistical Analysis}

Data from the three study datasets were analyzed separately. Prescription site was summarized according to the number of beds: hospital ( $\geq 20$ beds) and clinic ( $<20$ beds). Chronological changes in proportion of patients with two or fewer concomitant antidiabetic medications, proportion of patients using concomitant diuretics, and average daily doses of SU and insulin were aggregated quarterly by the index date.

A sensitivity analysis was conducted for the $\mathrm{H}$ - and P-datasets by including only patients with any record during the pre-index period to check for any potential biases due to nonavailability of continuous enrollment data in these datasets. All analyses were performed using SAS ${ }^{\circledR}$ Studio Release 3.5 (SAS Institute Inc., Cary, USA). JVS, TN, and TK had access to the database and all authors had access to the study results.

\section{RESULTS}

In total, 14,861 SGLT2i new users in the P-dataset, 27,039 in the H-dataset, and 12,408 in the I-dataset were included in this analysis (Fig. 1). Median age was 59 years in the P- and H-datasets and 52 years in the I-dataset, respectively. A median of two antidiabetic medications (by class) were prescribed during the pre-index period and at index (i.e., at SGLT2i initiation) (Table 1). Other patient characteristics have been previously described [31]. Overall, all three datasets showed similar results.

\section{Pre-Index and Concomitant Antidiabetic Medication}

The majority were taking one to three antidiabetic medications before initiating SGLT2i (Fig. 2a). The most frequently prescribed antidiabetic medications were DPP4i, followed 
by BG, and SU (Fig. 2b). Approximately 70\% were taking one to three concomitant antidiabetic medications at index (Fig. 3a). The proportion of patients taking two or fewer concomitant antidiabetic medications was observed to increase over time (Fig. 3b). Overall, DPP4i, BG, and SU were the most frequently prescribed concomitant antidiabetic medications in all datasets (Fig. 3c).

\section{Daily Dose of Glimepiride and Insulin at SGLT2i Add-On}

Among three subclasses of SU (glimepiride, glibenclamide, and gliclazide), glimepiride was most frequently prescribed to SGLT2i new users (P-dataset, 87.6\%; H-dataset, 88.6\%; I-dataset, $88.9 \%)$. Therefore, the results for glimepiride subcohorts are presented.

The average daily dose of glimepiride decreased from $2.1 \mathrm{mg} /$ day during the pre-index period to $1.8 \mathrm{mg}$ /day at SGLT2i add-on in the P-dataset and from 1.9 to $1.7 \mathrm{mg} /$ day in the both $\mathrm{H}$ - and I-datasets, respectively (Table 2 ). The daily glimepiride dose was reduced in approximately $20 \%$ of the co-users at SGLT2i add-on. Also, a decreasing trend was observed in the average daily dose of glimepiride at index date during the first 3 years of launch (Fig. 4a).

On the other hand, the average daily insulin dose (units) increased slightly at SGLT2i add-on, compared with the pre-index period (Table 3). However, in approximately $40 \%$ of insulin cousers, the daily insulin dose was reduced at the time of SGLT2i add-on compared with that in the pre-index period. The average daily insulin dose at SGLT2i add-on was higher during the first 15 months of launch and then decreased and became stable in the subsequent months (Fig. 4b).

\section{Concomitant Medications at SGLT2i Initiation}

SGLT2i new users in all datasets were taking a median of four to five concomitant medications (by MHLW classification) at index (Table 1). Nearly $40 \%$ or more patients in the datasets were taking at least five concomitant medications (Fig. 5a). Other than "agents affecting metabolism", which includes antidiabetic medications, cardiovascular agents were frequently co-prescribed (Fig. 5b).

Diuretics were co-prescribed in less than $10 \%$ of SGLT2i new users at index (P-dataset, 6.3\%; H-dataset, 9.0\%; and I-dataset, 3.0\%). Diuretics use increased slightly over time in the P- and $\mathrm{H}$-datasets, whereas no obvious changes were observed in the I-dataset (Fig. S1 in the supplementary material). Across the specialties, concomitant diuretics were most frequently prescribed by the cardiology department in all datasets (Fig. 6).

\section{Sensitivity Analysis}

Excluding patients without any pre-index record reduced the number of SGLT2i new users from 14,861 to 14,156 (ca. 5\%, P-dataset) and 27,039 to 26,594 (ca. $2 \%$, H-dataset), respectively (Table S1 in the supplementary material). Patient demographics and baseline characteristics for the full study cohort were described elsewhere [31]. The demographics of SGLT2i new users in the sensitivity cohort were similar to the full study cohort (Table S1 in the supplementary material). Comorbidities, complications, HbA1c levels, body weight, body mass index (BMI), and estimated glomerular filtration rate (eGFR) values were also similar among the full cohort and sensitivity cohort. The numbers of pre-index and concomitant antidiabetic medications prescribed to SGLT2i new users were also similar in both cohorts (Fig. S2 in the supplementary material).

\section{DISCUSSION}

The present analysis sought to examine the utilization patterns of SGLT2i in the first 3 years of their launch in Japan. The findings from this large-scale data analysis showed that SGLT2i were mostly used as second- or later-line antidiabetic medication, usually as an add-on to DPP4i, BG, and/or SU. SGLT2i were frequently used as part of a dual, triple, or quadruple regimen. For SGLT2i new users taking concomitant SU or insulin medications, SU and insulin doses 
Table 2 Characteristics of glimepiride subcohort at index

P-dataset $(N=3457) \quad$ H-dataset $(N=6808) \quad$ I-dataset $(N=2975)$

Age, years

\begin{tabular}{|c|c|c|c|}
\hline Mean (SD) & $59.9(12.0)$ & $59.1(11.7)$ & $52.7(8.2)$ \\
\hline Median (IQR) & $61(51-68)$ & $60(51-67)$ & $53(47-58)$ \\
\hline Gender, male, $n(\%)$ & $2207(63.8)$ & $4453(65.4)$ & $2254(75.8)$ \\
\hline \multicolumn{4}{|l|}{ Prescription site ${ }^{\mathrm{a}}, n(\%)$} \\
\hline Clinic ( $<20$ beds $)$ & $1516(43.9)$ & - & $2091(70.3)$ \\
\hline Hospital ( $\geq 20$ beds) & $1941(56.1)$ & $6808(100)$ & $884(29.7)$ \\
\hline \multicolumn{4}{|l|}{ Specialty, $n(\%)$} \\
\hline General internal medicine & $1777(51.4)$ & $4158(61.1)$ & - \\
\hline Diabetology $^{\mathrm{b}}$ & $117(3.4)$ & $1438(21.2)$ & - \\
\hline Cardiology & $133(3.8)$ & $601(8.8)$ & - \\
\hline Others & $427(12.4)$ & $471(6.9)$ & - \\
\hline Unknown specialties & $1001(29.0)$ & $140(2.1)$ & - \\
\hline Multiple specialties & $2(0.1)$ & - & - \\
\hline \multicolumn{4}{|l|}{ Daily glimepiride dose at pre-index, mg } \\
\hline Mean (SD) & $2.1(1.5)$ & $1.9(1.4)$ & $1.9(1.4)$ \\
\hline Median (IQR) & $2.0(1.0-3.0)$ & $1.5(1.0-2.3)$ & $1.5(1.0-2.2)$ \\
\hline \multicolumn{4}{|l|}{ Daily glimepiride dose at index, mg } \\
\hline Mean (SD) & $1.8(1.4)$ & $1.7(1.3)$ & $1.7(1.3)$ \\
\hline Median (IQR) & $1.0(1.0-2.0)$ & $1.0(1.0-2.0)$ & $1.0(1.0-2.0)$ \\
\hline Any glimepiride dose reduction at index, $n(\%)$ & $788(22.8)$ & $1350(19.8)$ & $653(21.9)$ \\
\hline
\end{tabular}

P-dataset: From a pharmacy claims database using data from over 800 pharmacies nationwide which provided a coverage of approximately $2 \%$ of all outpatient prescriptions

H-dataset: From a hospital-based administrative database constructed from data for inpatients and outpatients from 287 Diagnosis Procedure Combination (DPC) hospitals

I-dataset: From an insurance claims database containing medical and prescription claims of 3.8 million employees and their dependents that were mostly aged $\leq 65$ years

All results are at index unless otherwise stated $I Q R$ interquartile range, $S D$ standard deviation

${ }^{a}$ Number of patients who had their medication prescriptions fulfilled at a clinic ( $<20$ beds) or a hospital ( $\geq 20$ beds)

b Also includes endocrinology and metabolism

at SGLT2i add-on decreased slightly over the study period. SGLT2i new users were taking a number of other concomitant medications, of which cardiovascular agents were frequently prescribed.
The pre-index and concomitant medication utilization patterns identified in this study concur with the results of post-marketing surveillance of ipragliflozin, canagliflozin, or tofogliflozin in Japan [41-43], but this study 


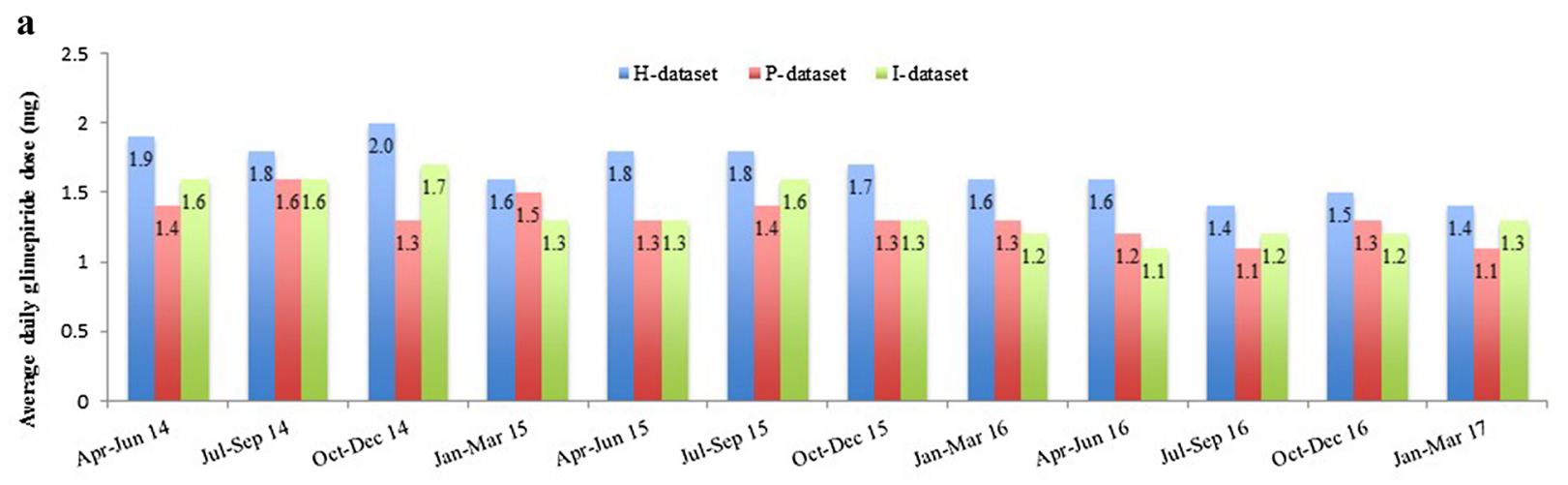

\begin{tabular}{|c|c|c|c|c|c|c|c|c|c|c|c|c|}
\hline & \begin{tabular}{|l|} 
Apr-Jun 14 \\
\end{tabular} & \begin{tabular}{|l|} 
Jul-Sep 14 \\
\end{tabular} & Oet-Dec 14 & Jan-Mar 15 & Apr-Jun 15 & \begin{tabular}{|l|} 
Jul-Sep 15 \\
\end{tabular} & Oct-Dec 15 & Jan-Mar 16 & Apr-Jun 16 & \begin{tabular}{|l|} 
Jul-Sep 16 \\
\end{tabular} & Oct-Dec 16 & Jan-Mar 17 \\
\hline H-dataset (n) & 618 & 813 & 629 & 534 & 1831 & 2325 & 3159 & 3444 & 3242 & 2876 & 3523 & 4045 \\
\hline P-dataset (n) & 1133 & 861 & 529 & 462 & 1117 & 1103 & 1445 & 1520 & 1593 & 1521 & 1744 & 1833 \\
\hline I-dataset (n) & 1162 & 715 & 449 & 370 & 1115 & 1154 & 1196 & 1239 & 1193 & 1187 & 1244 & 1384 \\
\hline
\end{tabular}

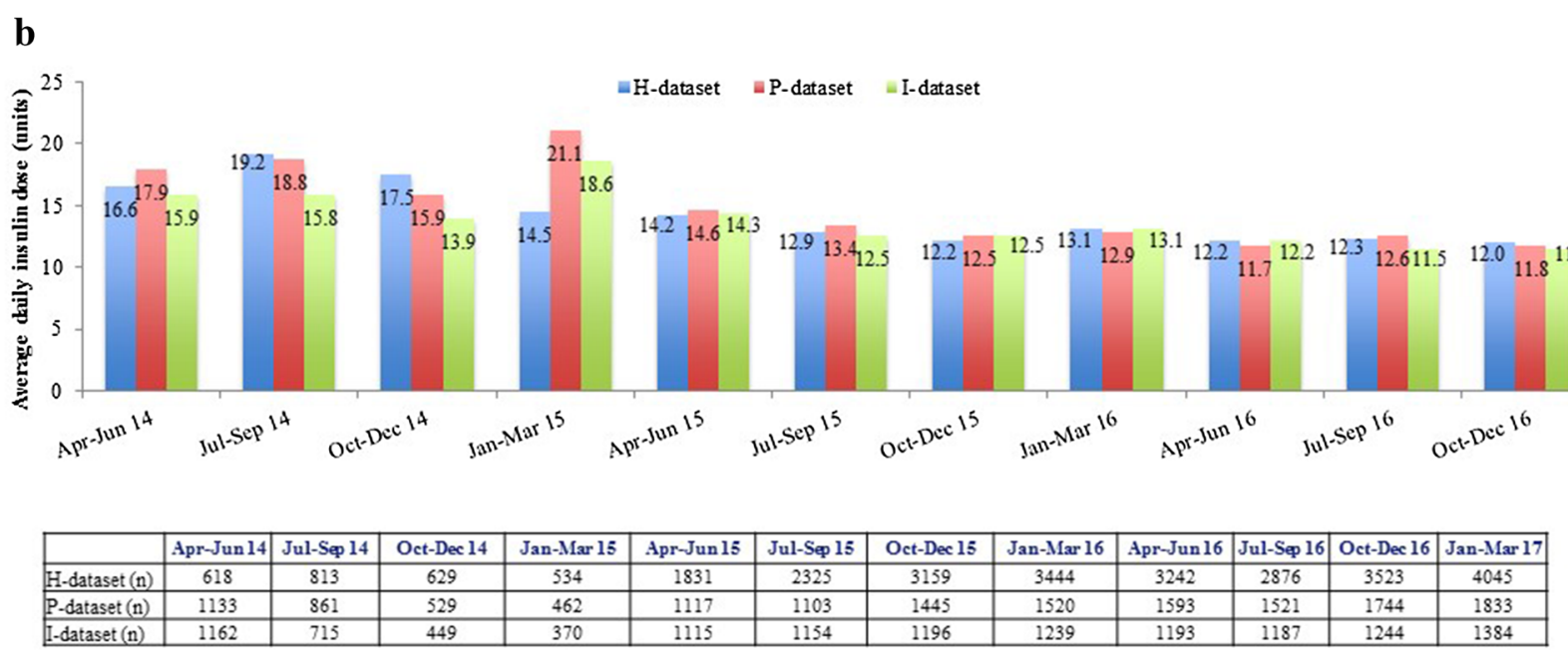

Fig. 4 Concomitant glimepiride and insulin dose. a Average daily glimepiride dose (mg) per quarter at SGLT2i addon. b Average daily insulin dose (units) per quarter at SGLT2i add-on. P-dataset: From a pharmacy claims database using data from over 800 pharmacies nationwide which provided a coverage of approximately $2 \%$ of all outpatient prescriptions. H-dataset: From a hospital-based administrative database constructed from data for

extends the findings of these studies by examining the prescribing trend following the launch of SGLT2i in Japan. The proportion of SGLT2i new users taking two or fewer concomitant antidiabetic medications at index increased over the study period, suggesting a trend towards prescribing fewer concomitant antidiabetic medications. Concomitant diuretic inpatients and outpatients from 287 Diagnosis Procedure Combination (DPC) hospitals. I-dataset: From an insurance claims database containing medical and prescription claims of 3.8 million employees and their dependents who were mostly aged 65 years or less. For insulin, the January to March 2017 data is not shown as data for the quarter after March 2017 needed to compute the average daily dose was not collected

use was generally low in SGLT2i users, consistent with the prescribing guidance provided in the package inserts for SGLT2i [19-24]. However, a slight increase in concomitant diuretic use over time was observed in the $\mathrm{H}$ - and P-datasets. This may probably be related to increased prescription from the cardiology 
Table 3 Characteristics of insulin subcohort at index

\begin{tabular}{|c|c|c|c|}
\hline & P-dataset $(N=1643)$ & H-dataset $(N=4005)$ & I-dataset $(N=1109)$ \\
\hline \multicolumn{4}{|l|}{ Age, years } \\
\hline Mean (SD) & $57.7(12.4)$ & $56.5(12.0)$ & $51.4(8.7)$ \\
\hline Median (IQR), years & $58(49-66)$ & $57(48-66)$ & $51(46-57)$ \\
\hline Gender, male, $n(\%)$ & $941(57.3)$ & $2198(54.9)$ & $755(68.1)$ \\
\hline \multicolumn{4}{|l|}{ Prescription site ${ }^{\mathrm{b}}, n(\%)$} \\
\hline Clinic ( $<20$ beds $)$ & $477(29.0)$ & - & $564(50.9)$ \\
\hline Hospital ( $\geq 20$ beds) & $1166(71.0)$ & $4005(100)$ & $545(49.1)$ \\
\hline \multicolumn{4}{|l|}{ Specialty, $n(\%)$} \\
\hline General internal medicine & $753(45.8)$ & $2492(62.2)$ & - \\
\hline Diabetology ${ }^{\mathrm{b}}$ & $126(7.7)$ & $1142(28.5)$ & - \\
\hline Cardiology & $34(2.1)$ & $118(2.9)$ & - \\
\hline Others & $153(9.3)$ & $205(5.1)$ & - \\
\hline Unknown specialties & $572(34.8)$ & $48(1.2)$ & - \\
\hline Multiple specialties & $5(0.3)$ & - & - \\
\hline \multicolumn{4}{|l|}{ Daily insulin dose (units) at pre-index } \\
\hline Mean (SD) & $12.7(8.1)$ & $12.0(8.1)$ & $12.4(6.0)$ \\
\hline Median (IQR) & $10.4(8.2-16.6)$ & $9.9(7.3-15.7)$ & $10.4(8.4-16.6)$ \\
\hline \multicolumn{4}{|l|}{ Daily insulin dose (units) at index } \\
\hline Mean (SD) & $13.8(8.7)$ & $13.1(8.3)$ & $13.5(8.4)$ \\
\hline Median (IQR) & $10.7(8.6-17.1)$ & $10.7(8.6-17.1)$ & $10.7(8.6-17.1)$ \\
\hline Any insulin reduction at index, $n(\%)$ & $619(37.7)$ & $1497(37.4)$ & $446(40.2)$ \\
\hline
\end{tabular}

P-dataset: From a pharmacy claims database using data from over 800 pharmacies nationwide which provided a coverage of approximately $2 \%$ of all outpatient prescriptions

H-dataset: From a hospital-based administrative database constructed from data for inpatients and outpatients from 287 Diagnosis Procedure Combination (DPC) hospitals

I-dataset: From an insurance claims database containing medical and prescription claims of 3.8 million employees and their dependents that were mostly aged $\leq 65$ years

All results are at index unless otherwise stated $I Q R$ interquartile range, $S D$ standard deviation

a Number of patients who had their medication prescriptions fulfilled at a clinic $(<20$ beds) or a hospital ( $\geq 20$ beds)

b Also includes endocrinology and metabolism

department reported in the first publication [31].

The risk of hypoglycemia may increase when SGLT2i is added to SU or insulin. Physicians are advised to reduce the dose of SU or insulin at
SGLT2i add-on and to make only small adjustments to the insulin dose to reduce the risk of ketoacidosis [19-25]. The average daily glimepiride dose decreased slightly at SGLT2i initiation and about one in five patients had their 

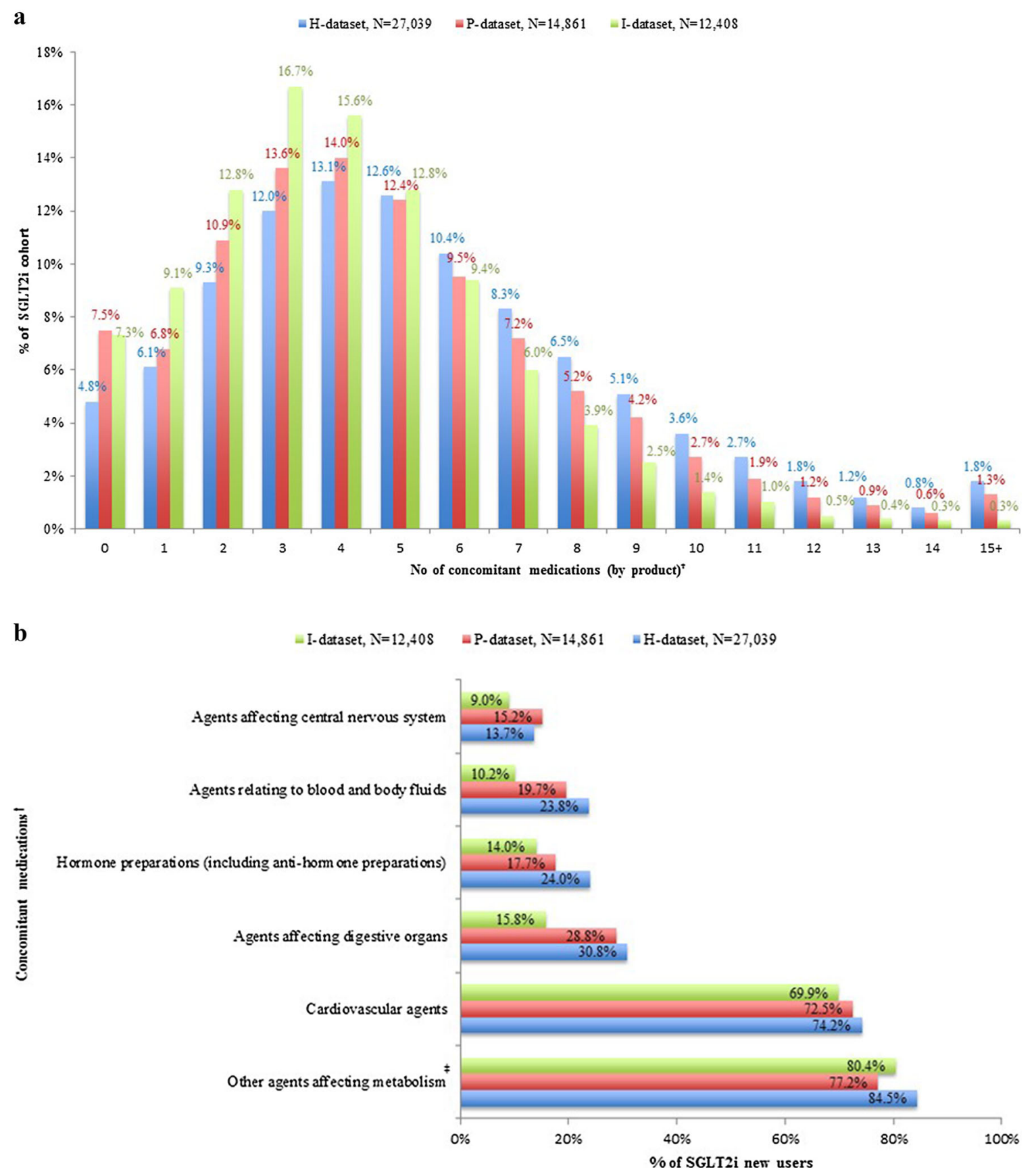

Fig. 5 Distribution of the number (a) and type (b) of concomitant medications. P-dataset: From a pharmacy claims database using data from over 800 pharmacies nationwide which provided a coverage of approximately $2 \%$ of all outpatient prescriptions. H-dataset: From a hospital-based administrative database constructed from data for inpatients and outpatients from 287 Diagnosis
Procedure Combination (DPC) hospitals. I-dataset: From an insurance claims database containing medical and prescription claims of 3.8 million employees and their dependents who were mostly aged 65 years or less. Dagger indicates data based on Ministry of Health, Labour and Welfare drug classification [40]. Double dagger indicates data include oral antidiabetic medications 
glimepiride dose reduced at SGLT2i add-on. Although the average daily insulin dose increased slightly by ca. 1 unit at SGLT2i addon, the daily insulin dose was reduced in two in five patients and the average daily dose at SGLT2i add-on decreased slightly 15 months after SGLT2i launch. These observations suggest a trend towards reducing concomitant SU or insulin doses in SGLT2i users over time.

Our findings should be interpreted within the context of the study limitations. The results of the I-datasets may not be generalizable to older diabetes populations because of the lack of patients over 75 years old. In addition, continuous enrollment information is not available for the P- and H-datasets to verify the index prescription. That is, out-of-panel pharmacy/ hospital prescription could not be obtained. Although we captured sufficient pre-index prescriptions, implying some follow-up ability of the patients within the panel pharmacies and hospitals, the prescriptions for pre-index and concomitant medications may be underestimated in the P- and $\mathrm{H}$-datasets. We explored the number of prescription sites per patient during the pre-index period and at the index date in the I-dataset and found that $95.6 \%(10,490$ out of 10,968 with pre-index antidiabetic medications) of the SGLT2i new users received their diabetes treatment at a single site during the 6-month pre-index period. At the index date, more than $99.9 \%(12,403 /$ 12,408 ) of the SGLT2i new users received their concomitant antidiabetic medications from one site and $96.9 \%(12,020 / 12,408)$ who received any concomitant medication were also from a single site. Therefore, we believe that the majority of patients received their diabetes treatment at a single site during the 6-month pre-index period and on the index date. Although we do not have data on the number of pharmacies where the prescription items were dispensed on the same day, we assumed that the impact of not capturing prescriptions from outof-panel pharmacy/hospitals may be limited.

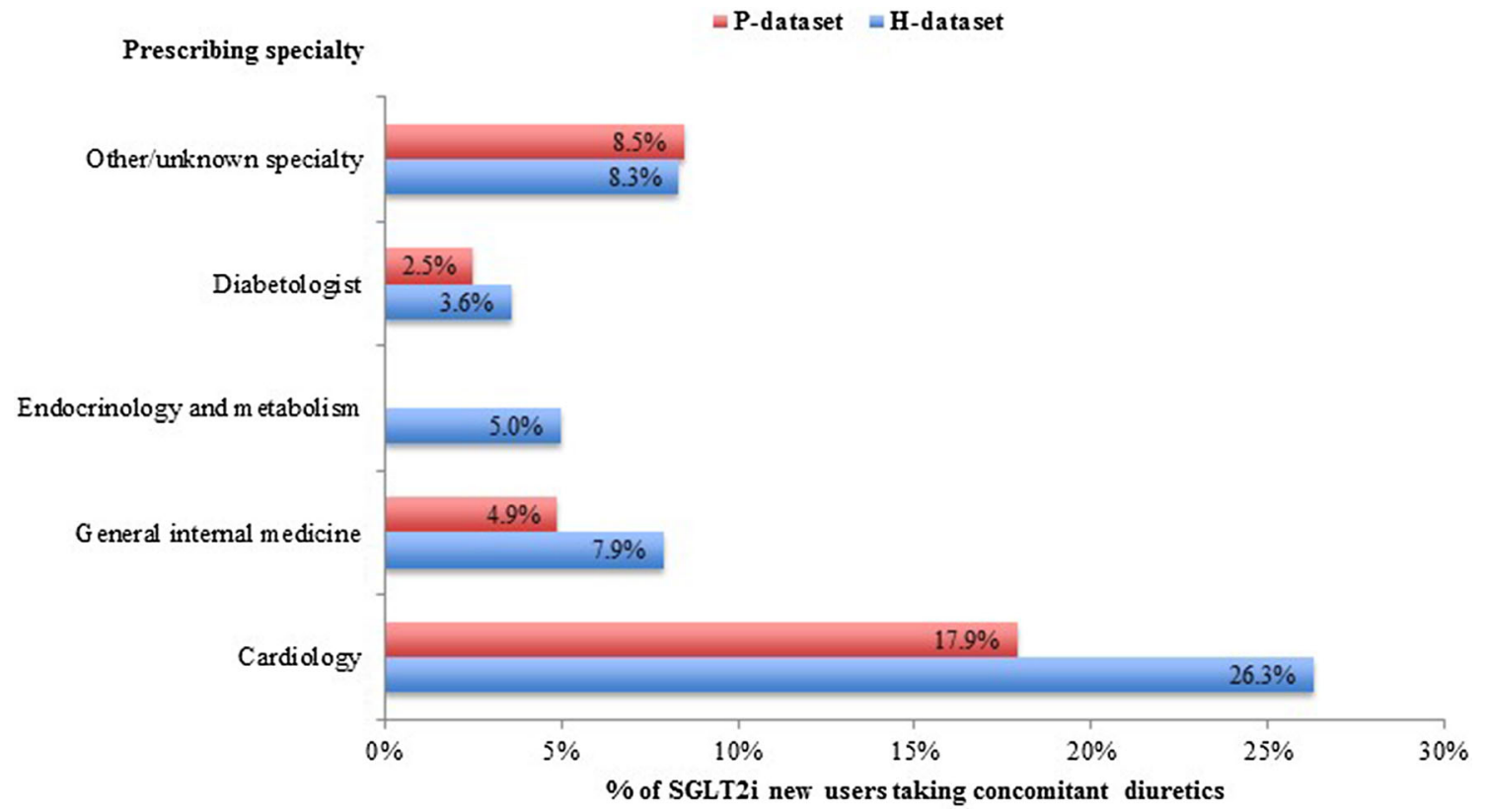

Fig. 6 Distribution of concomitant diuretics use in SGLT2i new users by prescriber specialty. P-dataset: From a pharmacy claims database using data from over 800 pharmacies nationwide which provided a coverage of approximately $2 \%$ of all outpatient prescriptions. H-dataset: From a hospital-based administrative database constructed from data for inpatients and outpatients from 287 Diagnosis Procedure Combination (DPC) hospitals. Data on prescribing specialty were not available for the I-dataset. Endocrinology and metabolism category did not exist in the P-dataset 
We did not place additional restrictions on the $\mathrm{H}$ - and P-datasets (such as having any prescription at least once in 3 months as a proxy of continuous enrollment) as this may select patients who were more "loyal" to a pharmacy or hospital, or who were sicker, and thus visited the pharmacy or hospital more frequently [30]. We conducted a sensitivity analysis using only data from patients who had any record during the pre-index period. The results revealed no difference when compared with the study cohort, suggesting that this did not affect the results of this analysis.

Also, hospitalized patients at the index date were excluded from analysis. Our initial intention was to include both inpatients and outpatients but because of the limited follow-up ability, unexpectedly large proportions of patients in the H-dataset were identified as inpatients at the index date. Although this proportion was smaller in SGLT2i new users (13\%) compared with the new users of other OADs (45\%) [31], we decided to exclude inpatients at index date because we believed it is unlikely that patients with diabetes started their OAD medications at an inpatient setting. Rather, the "index date" identified in this study was the first date where patients appeared in the hospital database with the OAD prescription. Indeed, inpatients in the I-dataset (where patients can be followed up) was limited to $2.1 \%$. Even considering the difference in age distribution between the $\mathrm{H}$ - and I-datasets, we believe that the proportion of inpatients should not be that high. Thus, we excluded inpatients from the analysis for better generalizability.

As in the other database studies, we do not know if the patients actually took the prescribed medications as recorded. Further, some prescriptions could have been changed or cancelled at dispensing. In addition, the actual daily insulin dose was not available in the data sources and therefore estimated using the average during the pre- or post-index period. Thus, it may not reflect the prescribers' intention correctly. Despite these limitations, the results were generally consistent between the three datasets and provide a real-world snapshot of the utilization pattern shortly after the launch of SGLT2i in Japan.

\section{CONCLUSION}

This study characterized the drug utilization patterns in patients initiating SGLT2 $\mathrm{i}$ in the first 3 years of its launch in Japan. SGLT2i were frequently used as second- or later-line treatment and as part of a dual, triple, or quadruple regimen, and were co-prescribed with many other agents, predominantly cardiovascular medications. DPP4i, BG, and/or SU were frequently used before and at SGLT2i initiation. For SGLT2i users taking concomitant SU or insulin medications, the daily doses of SU and insulin at SGLT2i add-on were gradually reduced over time.

\section{ACKNOWLEDGEMENTS}

Funding. Sponsorship for this study and the Rapid Service Fee were funded by Astellas Pharma Inc., Tokyo, Japan.

Medical Writing and Editorial Assistance. Medical writing and editorial support was provided by Hui Hwa Choo of Tech Observer Asia Pacific Pte. Ltd. and was funded by Astellas Pharma Inc., Japan

Authorship. All named authors meet the International Committee of Medical Journal Editors (ICMJE) criteria for authorship for this article, take responsibility for the integrity of the work as a whole, and have given their approval for this version to be published.

Disclosures. James Van Schyndle is an employee of Astellas US, LLC. Yuichiro Ito is an employee of Astellas Pharma Inc., Japan. Takuya Nishimura is an employee of Astellas Pharma Inc., Japan. Toshifumi Sugitani is an employee of Astellas Pharma Inc., Japan. Tomomi Kimura is an employee of Astellas Pharma Inc., Japan.

Compliance with Ethics Guidelines. The study protocol was approved by the Medical Affairs Japan Protocol Review Committee on 22 June 2017 before study commencement. As the 
study only involved analysis of pre-existing data from the data sources and the data were anonymized, written informed consent was not required, according to applicable regulations in Japan [32, 33]. This study was conducted in accordance with the guidelines for Good Pharmacoepidemiology Practice [34].

Data Availability. Access to anonymized individual participant level data will not be provided for this trial as it meets one or more of the exceptions described on www. clinicalstudydatarequest.com under "Sponsor Specific Details for Astellas".

Open Access. This article is distributed under the terms of the Creative Commons Attribution-NonCommercial 4.0 International License (http://creativecommons.org/licenses/ by-nc/4.0/), which permits any noncommercial use, distribution, and reproduction in any medium, provided you give appropriate credit to the original author(s) and the source, provide a link to the Creative Commons license, and indicate if changes were made.

\section{REFERENCES}

1. Charvat H, Goto A, Goto M, et al. Impact of population aging on trends in diabetes prevalence: a meta-regression analysis of 160,000 Japanese adults. J Diabetes Investig. 2015;6:533-42.

2. Zheng Y, Ley SH, Hu FB. Global aetiology and epidemiology of type 2 diabetes mellitus and its complications. Nat Rev Endocrinol. 2018;14:88-98.

3. Chao EC, Henry RR. SGLT2 inhibition-a novel strategy for diabetes treatment. Nat Rev Drug Discov. 2010;9:551-9.

4. Cersosimo E, Solis-Herrera C, Triplitt C. Inhibition of renal glucose reabsorption as a novel treatment for diabetes patients. J Bras Nefrol. 2014;36:80-92.

5. Wilding JP. The role of the kidneys in glucose homeostasis in type 2 diabetes: clinical implications and therapeutic significance through sodium glucose co-transporter 2 inhibitors. Metabolism. 2014;63:1228-37.

6. Araki E, Tanizawa Y, Tanaka Y, et al. Long-term treatment with empagliflozin as add-on to oral antidiabetes therapy in Japanese patients with type 2 diabetes mellitus. Diabetes Obes Metab. $2015 ; 17: 665-74$.

7. Inagaki N, Kondo K, Yoshinari T, Kuki H. Efficacy and safety of canagliflozin alone or as add-on to other oral antihyperglycemic drugs in Japanese patients with type 2 diabetes: a 52-week open-label study. J Diabetes Investig. 2015;6:210-8.

8. Kadowaki T, Haneda M, Inagaki N, et al. Efficacy and safety of empagliflozin monotherapy for 52 weeks in Japanese patients with type 2 diabetes: a randomized, double-blind, parallel-group study. Adv Ther. 2015;32:306-18.

9. Kaku K, Maegawa H, Tanizawa Y, et al. Dapagliflozin as monotherapy or combination therapy in Japanese patients with type 2 diabetes: an open-label study. Diabetes Ther. 2014;5:415-33.

10. Kashiwagi A, Kazuta K, Goto K, Yoshida S, Ueyama E, Utsuno A. Ipragliflozin in combination with metformin for the treatment of Japanese patients with type 2 diabetes: ILLUMINATE, a randomized, double-blind, placebo-controlled study. Diabetes Obes Metab. 2015;17:304-8.

11. Kashiwagi A, Kazuta K, Yoshida S, Nagase I. Randomized, placebo-controlled, double-blind glycemic control trial of novel sodium-dependent glucose cotransporter 2 inhibitor ipragliflozin in Japanese patients with type 2 diabetes mellitus. J Diabetes Investig. 2014;5:382-91.

12. Seino $\mathrm{Y}$, Inagaki $\mathrm{N}$, Haneda $\mathrm{M}$, et al. Efficacy and safety of luseogliflozin added to various oral antidiabetic drugs in Japanese patients with type 2 diabetes mellitus. J Diabetes Investig. 2015;6:443-53.

13. Seino Y, Sasaki T, Fukatsu A, Ubukata M, Sakai S, Samukawa Y. Efficacy and safety of luseogliflozin as monotherapy in Japanese patients with type 2 diabetes mellitus: a randomized, double-blind, placebo-controlled, phase 3 study. Curr Med Res Opin. 2014;30:1245-55.

14. Tanizawa Y, Kaku K, Araki E, et al. Long-term safety and efficacy of tofogliflozin, a selective inhibitor of sodium-glucose cotransporter 2, as monotherapy or in combination with other oral antidiabetic agents in Japanese patients with type 2 diabetes mellitus: multicenter, open-label, randomized controlled trials. Expert Opin Pharmacother. 2014;15:749-66.

15. Zelniker TA, Wiviott SD, Raz I, et al. SGLT2 inhibitors for primary and secondary prevention of cardiovascular and renal outcomes in type 2 diabetes: a systematic review and meta-analysis of cardiovascular outcome trials. Lancet. 2019;393:31-9. 
16. American Diabetes Association. Pharmacologic approaches to glycemic treatment: standards of medical care in diabetes-2019. Diabetes Care. 2019;42:S90-102.

17. Davies MJ, D'Alessio DA, Fradkin J, et al. Management of hyperglycemia in type 2 diabetes, 2018. A consensus report by the American Diabetes Association (ADA) and the European Association for the Study of Diabetes (EASD). Diabetes Care. 2018;2018(41):2669-701.

18. Haneda $M$, Noda $M$, Origasa $H$, et al. Japanese clinical practice guideline for diabetes 2016. J Diabetes Investig. 2018;9:657-97.

19. Ipragliflozin package insert. Pharmaceuticals and Medical Devices Agency. 2019. http://www.info. pmda.go.jp/go/pack/3969018F1022_1_11/. Accessed 20 June 2019.

20. Dapagliflozin package insert. Pharmaceuticals and Medical Devices Agency. 2019. http://www.info. pmda.go.jp/go/pack/3969019F1027_2_08/. Accessed 20 June 2019.

21. Canagliflozin package insert. Pharmaceuticals and Medical Devices Agency. 2019. http://www.info. pmda.go.jp/go/pack/3969022F1029_1_13/. Accessed 20 June 2019.

22. Empagliflozin package insert. Pharmaceuticals and Medical Devices Agency. 2019. http://www.info. pmda.go.jp/go/pack/3969023F1023_1_10/. Accessed 20 June 2019.

23. Tofogliflozin package insert. Pharmaceuticals and Medical Devices Agency. 2019. http://www.info. pmda.go.jp/go/pack/3969021F1032_1_10/. Accessed 20 June 2019.

24. Luseogliflozin package insert. Pharmaceuticals and Medical Devices Agency. 2019. http://www.info. pmda.go.jp/go/pack/3969020F1020_2_11/. Accessed 20 June 2019.

25. Fitchett D. A safety update on sodium glucose cotransporter 2 inhibitors. Diabetes Obes Metab. 2019;21:34-42. https://doi.org/10.1111/dom. 13611.

26. Kohro T, Yamazaki $\mathrm{T}$, Sato $\mathrm{H}$, et al. Trends in antidiabetic prescription patterns in Japan from 2005 to 2011. Int Heart J. 2013;54:93-7.

27. Oishi M, Yamazaki K, Okuguchi F, Sugimoto H, Kanatsuka A, Kashiwagi A. Changes in oral antidiabetic prescriptions and improved glycemic control during the years 2002-2011 in Japan (JDDM32). J Diabetes Investig. 2014;5:581-7.
28. Tanabe M, Motonaga R, Terawaki Y, Nomiyama T, Yanase T. Prescription of oral hypoglycemic agents for patients with type 2 diabetes mellitus: a retrospective cohort study using a Japanese hospital database. J Diabetes Investig. 2017;8:227-34.

29. Kadowaki T, Sarai N, Hirakawa T, Taki K, Iwasaki K, Urushihara H. Persistence of oral antidiabetic treatment for type 2 diabetes characterized by drug class, patient characteristics and severity of renal impairment: a Japanese database analysis. Diabetes Obes Metab. 2018;20:2830-9.

30. Nishimura R, Kato H, Kisanuki K, et al. Treatment patterns, persistence and adherence rates in patients with type 2 diabetes mellitus in Japan: a claims-based cohort study. BMJ Open. 2019;9:e025806.

31. Ito Y, Van Schyndle J, Nishimura T, Sugitani T, Kimura T. Characteristics of patients with diabetes initiating sodium glucose co-transporter-2 inhibitors (SGLT2i): real-world results from three administrative databases in Japan. Diabetes Ther. 2019;10:549-62.

32. Personal information protection act. Ministry of Justice Japan. 2015. http://www.japaneselaw translation.go.jp/law/detail/?printID=\&id=2781\&re $=£ v m=03$. Accessed 31 May 2019.

33. Anonymously Processed Information. Personal Information Protection Commission Secretariat, Japan. 2017. https://www.ppc.go.jp/files/pdf/The_ PPC_Secretariat_Report_on_Anonymously_Process ed_Information.pdf. Accessed 31 May 2019.

34. Public Policy Committee ISoP. Guidelines for good pharmacoepidemiology practice (GPP). Pharmacoepidemiol Drug Saf. 2016;25:2-10.

35. Medi-Trend (Out-of-hospital prescription database system). Kyowa Kikaku Ltd. 2014. https://www.kkkyowa.co.jp/medical_prescription.html. Accessed 22 July 2019.

36. Introducing MDV Database. Medical Data Vision Co., Ltd. https://www.mdv.co.jp/solution/ pharmaceutical/english/. Accessed 22 July 2019.

37. JMDC insurance database. JMDC Inc. https://www. jmdc.co.jp/en/. Accessed 31 May 2019.

38. Kimura S, Sato T, Ikeda S, Noda M, Nakayama T. Development of a database of health insurance claims: standardization of disease classifications and anonymous record linkage. J Epidemiol. 2010;20:413-9.

39. Databases available for pharmacoepidemiology researches in Japan. Japanese Society for Pharmacoepidemiology. 2018. http://www.jspe.jp/mt- 
static/FileUpload/files/JSPE_DB_TF_E.PDF. Accessed 31 May 2019.

40. Drug classification Ministry of Health, Labour and Welfare (MHLW). 2015. Revised 5th edition. https://www.mhlw.go.jp/file/05-Shingikai-124040 00-Hokenkyoku-Iryouka/0000084745.pdf. Accessed 22 July 2019.

41. Goda M, Yamakura T, Sasaki K, Tajima T, Ueno M. Safety and efficacy of canagliflozin in elderly patients with type 2 diabetes mellitus: a 1-year postmarketing surveillance in Japan. Curr Med Res Opin. 2018;34:319-27.
42. Nakamura I, Maegawa $\mathrm{H}$, Tobe $\mathrm{K}$, Tabuchi $\mathrm{H}$, Uno S. Safety and efficacy of ipragliflozin in Japanese patients with type 2 diabetes in real-world clinical practice: interim results of the STELLA-LONG TERM post-marketing surveillance study. Expert Opin Pharmacother. 2018;19:189-201.

43. Utsunomiya K, Shimmoto N, Senda M, et al. Safety and effectiveness of tofogliflozin in elderly Japanese patients with type 2 diabetes mellitus: a post-marketing study (J-STEP/EL Study). J Diabetes Investig. 2017;8:766-75. 